\section{RECENT DISCOVERIES IN THE PLANET MARS}

$\mathrm{A}^{\mathrm{N}}$ intended article, of which an announcement appeared in NATURE a few weeks back, on the topography of Mars as delineated for the second time by Prof. Schiaparelli at Milan during the opposition of $1879-80$, has been anticipated and in "part superseded by informa tion which has been received relative to the more recent discoveries made by him in the beginning of the present year. Pending the preparation of a fuller and more detailed memoir, he has published a preliminary notice, read before the Academia dei Lincei on March 5, and accompanied by a photographed drawing of the planet's surface. The results are of a very remarkable and unexpected character; and as through the courtesy of this distinguished observer, the notice and photograph have been placed in my hands, I am induced to reproduce the latter, which, though not pretending to minute accuracy (the original, in fact, is only a provisional sketch), will give a sufficient idea of the marvellous duplication of the so-called "canals," which, between
January 19 and February 24, in about twenty instances, unfolded itself progressively under the observer's eye. The discussion which took place at the late meeting of the Astronomical Society, so far as my information extends, substantiated strongly by independent evidence the existence of these long, narrow streaks, some of them even in positions where they have not been delineated by Schiaparelli; but their duplication by similar and parallel lines does not seem to have been elsewhere noticed. Some difference of opinion may possibly be expected concerning these strange appearances; and the consequent enfeebling (to say the least of it) of the longadmitted terrestrial analogy may be, to some minds, unacceptable; but the established reputation of the observer demands at any rate a respectful attention to his statements. It may be preferable to suspend a more detailed account till we receive a full elucidation of the subject in the memoir, of which we possess only a preliminary notice; for the present it may suffice to mention that he found the atmosphere of Mars apparently clearer than in 1877 , and was thus enabled to recover the markings then detected more satisfactorily

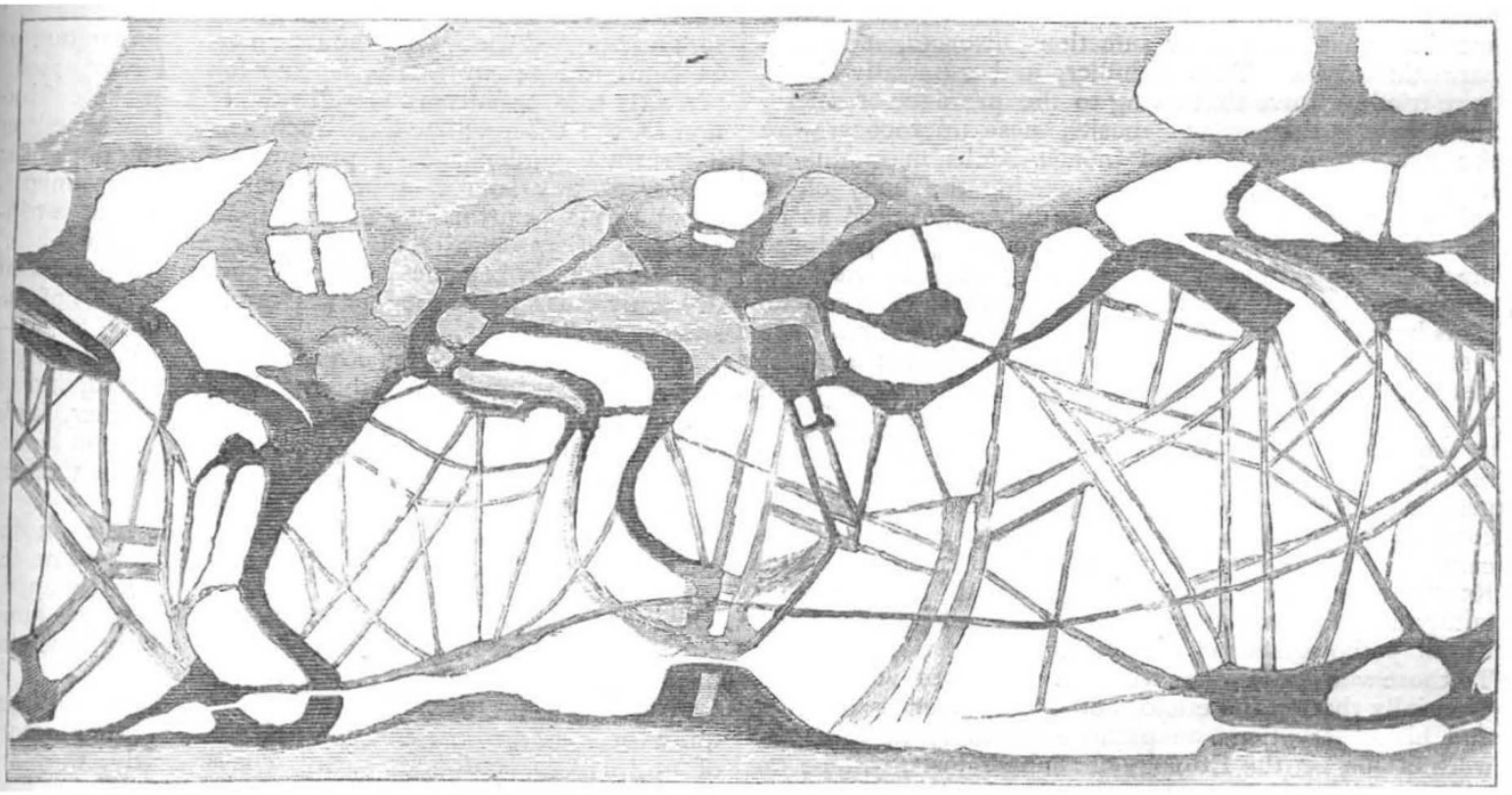

even than in $1879-80$, and to confirm the general accuracy of his two earlier charts; while the concise, but very clear intimations that he has given, as to the variable brightness of some great regions, the progressive enlargement on one side since I879 of the "Kaiser Sea" (his Syrtis Magna), the brightening of certain supposed continents or islands towards the limbs, the confirmed existence of oblique white streaks, the unfolding of minute labyrinthine detail, and the continuous development already mentioned, day after day, of the collateral lines which double the socalled "canals," and extend with them ordinarily along great circles of the sphere-all these and similar announcements make us anxiously desire a more extended and detailed communication. For some of these most remarkable appearances parallels may be to a certain extent produced from the results of earlier observers; but, so far as at present appears, the duplication stands alone. The discoverer is disposed to infer a connection between these progressive developments and the seasons of the planet, and on that account hopes that, owing to the position of the axis at the ensuing opposition at the opening of I884, notwithstanding, the diminished diameter (only I $2^{\prime \prime} \cdot 9$ ), confirmation of his announcements may be obtained from other observers. We sincerely trust that a report which has reached us may be verified as to the erection of a much larger telescope in the Royal Observatory at Milan, and that the extraordinary talent and diligence of the director may be richly rewarded, not only by the confirmation but the extension of results which must so materially influence our conclusions as to the physical condition of this peculiarly interesting planet. T. W. WEBB

\section{THE CAUSE OF TUBERCULOSIS}

THE first step in the rational treatment of every malady is obviously the recognition of its cause, and it is this recognition which enables medical men to do battle against disease. It is a truism to say that as regards infectious maladies the knowledge of their cause is an essential step in preventing their spread and arresting their ravages. The malady known as tuberculosis, and generally characterised by constitutional disturbance associated with the production of minute nodular newgrowths in the various organs, especially the lungs, spleen, lymphatic glands, serous membranes, the membranes of the brain, liver, \&c.-[at first greyish and transparent, but 
afterwards becoming opac and degenerating into a yellowish-looking débris, and hereby implicating and destroying the organs in which they are located]-has been shown to be an infectious malady communicable from one human being to another, from man to animal, and from animal to animal.

The successful experiments of inoculating with, feeding on, and causing to inhale human tubercular matter, carried out on the lower animals, such as guinea-pigs, rabbits, dogs, pigs, \&c., by Villemin, Dr. Wilson Fox, Mr. John Simon, and Dr. Burdon Sanderson, but especially by Cohnheim and Salomonsen, Tappeiner, and Baumgarten are conclusive in these respects. Similarly it has been shown that the tuberculosis of cattle or Perlsucht is communicable not only within the species but also to other animals. Whether Perlsucht is also communicable to man, especially through meat and milk, as is maintained by some observers (Semmer, Baumgarten, and others), is as yet an open question, and, as must be obvious to every one, one to which fearful importance is attached, considering how great the distribution of this disease is in the bovine species. What the cause of the malady is, has until now been undetermined, although it has been at various times surmised that, like other infectious diseases, it is of a parasitic origin. Thus Schüller, and especially Klebs, have tried to prove that owing to the presence of micrococci in the tubercular deposits, these micrococci were the materies morbi. And indeed Klebs maintains to have succeeded in cultivating outside the body of an animal, i.e. artificially the "monas tuberculosum," as he calls the said micrococcus, and to have again produced the tubercular disease by inoculating animals with this purified micrococcus. Klebs' observations and conclusions have not been accepted as reliable, and it has been reserved for Dr. Koch to discover the real cause of the disease, in identifying it with a specific bacillus. In a weighty paper, "The Etiology of Tuberculosis," published in the Berlin Klin. Wochenschr, 1882, No. I5, Dr. Koch sets forth the whole course of his investigation, the methods and experiments, all his observations and definite conclusions on this question, and anyone who peruses carefully this paper will come to the conclusion that Koch's results are to be accepted with unconditional faith, and I have no manner of doubt will be considered by all pathologists as of the very highest importance. To those who are familiar with Dr. Koch's previous work, especially that on the etiology of splenic fever or Anthrax, and his observations on pathogenic Bacteria, this last work of his, on the Etiology of Tuberculosis, will be an additional and brilliant testimony to his ingenious and successful method of research.

The first step in the inquiry was to ascertain whether any definite form of microphyte is constantly present in the tubercular deposits. This question could not be solved by the ordinary methods of research, but with new methods; it was decided in the affirmative. For the demonstration of the presence of the specific bacilluswhich Koch calls the tubercle-bacillus-the following method proved successful: Tubercular deposit fresh, or after hardening with reagents, is stained for twenty to twenty-four hours - at a temperature of 40 Centigrades, only half to one hour is required-with a half per cent. solution of methylene blue, to which a small quantity of a ro per cent. solution of caustic potash is added. After this, the tubercular material is stained for a minute or two in a concentrated watery solution of vesuvin, and then washed in distilled water. When examined under the microscope, all elements of the tubercular deposit, such as cells, nuclei, fibres, and granules, appear of a brownish colour, while the tubercle-bacilli alone stand out very conspicuously in a beautiful blue tint.

By this method Koch ascertained the constant presence of the specific bacillus in the tubercular eruption in man and animals, including the Perlsucht of cattle, both in spontaneous tuberculosis, as well as artificially produced, i.e. by inoculation. These bacilli differ from all other micro-organisms by characteristic properties.

The next step in the inquiry was one of essential importance in determining the nature of the bacillus as the materies morbi, viz. to isolate by successive cultivations outside the animal body, the tubercle-bacilli, and having thus completely freed them of all parts of tissue of the tubercular deposit to introduce them into the system of suitable animals. If these animals became afflicted with typical tuberculosis, and if at the same time similar animals kept under precisely the same conditions, but not infected with the bacilli, remained perfectly normal, it will be admitted that the exact proof has been given that the bacilli constitute the cause of the tubercular malady.

All these conditions have been fulfilled by Dr. Koch in an eminent degree.

The tubercle-bacilli were successfully cultivated outside the body of an animal. Pure serum of blood of sheep or cattle is sterilised by keeping it exposed in testtubes plugged with cotton wool, for six days daily for one hour, to a temperature of 58 centigrades. After this the serum is heated for several hours up to a temperature of 65 centigrades; by this it is transformed into a solid perfectly transparent mass, well adapted for the cultivation of the tubercle-bacilli. Such serum inoculated on its surface under special precautions with tubsrcular matter of any source-tuberculosis of man or animal, spontaneous or artificially produced, and kept at a temperature of 37 or 38 centigrades (i.e. about blood-heat) for over a week, becomes gradually covered with peculiar dry scaly masses; these masses are the colonies of the specific tubercle-bacillus.

A minute particle of this crop is used for establishing a second similar cultivation, this again for a third, and so on. Tubercle-bacilli obtained in this manner, after several successive generations, prove as effective in inoculating animals with typical tuberculosis as fresh tubercular matter.

All animals susceptible to the malady that Koch inoculated with these artificially cultivated bacilli, became invariably affected with the disease; not one escaped; while other similar animals kept under precisely the same conditions, except that they did not receive any tuberclebacilli, remained perfectly healthy.

It is important to notice that the tubercle-bacilli require for their growth and multiplication a temperature of at least 30 centigrades, and, consequently, they are limited to the animal body, unlike the bacillus that produces splenic fever or anthrax, which is capable of multiplication at ordinary temperatures, as low as 20 centigrades, and even less.

The expectorated matter of tubercular patients is generally charged with tubercle-bacilli, and these often contain spores. And it is probably through the presence of such spores that that matter retains for a long time its infective properties. Koch made experiments on guinea-pigs with such sputa after having been kept dry for fourteen days, for four weeks, and for eight weeks, and he found that in all instances the sputa had retained their full virulence. It is therefore just to assume that such sputa, even when dried on linen, clothes, or even distributed with the dust of the room, may be a source of infection.

The practical importance of the discoveries of Koch must be patent to everybody. In the recognition of the tubercle-bacilli as the fons et origo of this terrible pest of the human race-according to statistics quoted by Koch one-seventh of all deaths being caused by tuberculosisin the recognition of the presence of these bacilli in the sputa of tubercular patients, and in the tubercular deposits of cattle afflicted with Perlsucht, we have at once become supplied with the knowledge of the most common manner of how tuberculosis may and probably is spread, as well 
as with the weapon of combating the most fertile sources of infection. In preventing the distribution-either by proper disinfection, or by destruction - of the expectorations of tubercular persons, and further, in superintending and restricting the use of tubercular animals of the bovine species, we possess the means of preventing the spread of this deadly and terrible plague, and hereby saving a vast amount of human life. These discoveries of Dr. Koch were made entirely through experiments on living animals.

E. KLEIN

\section{DEEP.SEA EXPLORATION}

IN NATURE, vol. xviii. p. 348, we described Negretti and Zambra's Patent Deep-Sea Standard Thermometer. Some uncertainty as to the accuracy of its indications in deep sea service led to a re-arrangement of the instrument, which now greatly increases its reliability. The improvement is chiefly due to suggestions furnished by Commander Magraghi (see NATURE, vol. xxiv. p. 505)

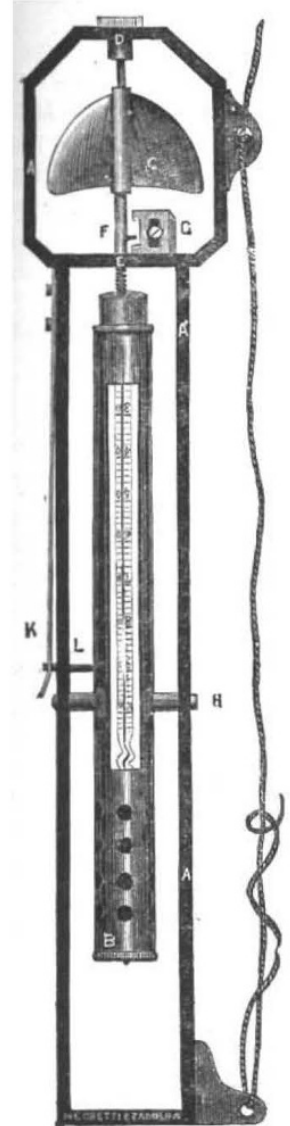

Fig. I.

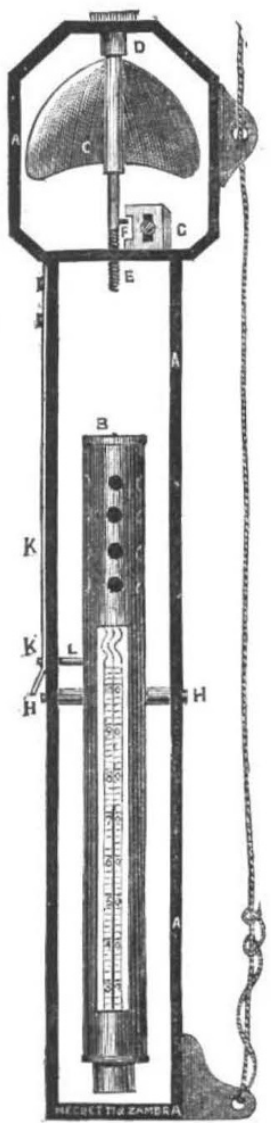

FIG. 2.

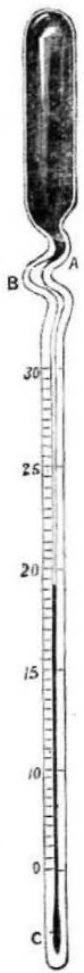

FIG. 3. (of the Royal Italian Navy) to Negretti and Zambra. Several of these improved thermometers may now be fastened on one line, and serial temperatures at any required depth obtained with certainty.

The woodcuts exhibit the apparatus, Fig. I, as prepared'for lowering down into the Sea, and Fig. 2 after the hauling up has commenced-the thermometer having reversed and registered the temperature at the moment of turning over. Fig. 3 shows the peculiar construction of Negretti and Zambra's inverted thermometer used in their improved deep-sea apparatus. The apparatus will be understood by reference to figures (Nos. I and 2). A is a metal frame, in which $B$, the thermometer, is pivoted upon an axis, H, but not balanced upon it. C is a screw- fan attached to a spindle, one end of which works in a socket, D, and at the other end is a screw, E, about half an inch long, and just above it is a small pin, F. On the spindle $\mathrm{G}$, is a sliding stop-piece, against which the pin, $\mathrm{F}$, impinges when the thermometer is adjusted for. use. The screw, $\mathrm{E}$, works into the end of the case, $\mathrm{B}$, the length of play to which it is adjusted. The number of turns of the screw entering the case is regulated by means of the pin, F, and stop-piece, G. The thermometer and its case is held in position by the screw, E, and descends into the sea in this position-as Fig. I ; the fan, C, not acting during the descent, because it is checked by the stop, $F$. When the ascent commences, the fan revolves, raises the screw, $\mathrm{E}$, and releases the thermometer, which then turns over and registers the temperature at that spot. When the hauling-up has caused the thermometer to turn over, a spring at $\mathrm{K}$ forces the pin, $\mathrm{L}$, into a slot in the case B, and clamps it (as seen in Fig. 2) until it is received on board, so that no change of position can occur during the ascent from any cause. The case, $\mathrm{B}$, is cut open to expose the scale of the thermometer, and also perforated to allow free passage of the water.

\section{SOME PRIMITIVE IDEAS ON METEOROLOGY}

$\mathrm{I}$ an article published in NATURE (vol. xxv. p. 82) on

the opinions of the Chinese Emperor Khang-hi on certain natural phenomena, it will be remembered that the yang and yin, or the male and female principles of Chinese philosophy, played a conspicuous part. Japan, it is well known, adopted at a very early period in its history the law, polity, science, philosophy, and writing of the Chinese, and with them the yang and yin; and it may not be uninteresting to our readers to see how the doctrine of these dual forces, mutually repellent as well as attractive, has been employed to explain the facts of meteorology. A recent issue of the Japan Gazette newspaper of Yokohama contains the translation of a work written in 182 I by a certain Arai Yoshinari, called the "Ten-chi-jii; or, Ideas about Heaven and Earth." The heavens, the writer says, are very high, the earth is very thick; we cannot ascend to the one or go down into the other; consequently man was unable for many generations to comprehend the phenomena of either ; but now the opinions of all philosophers on this subject are based on the action and reaction of the male and female, the active and passive principles of nature upon each other. The rain is a changed form of the male, and the vapour under the earth of the female principle. When the male principle sinks into the earth it pursues the female. The earth is the mother of all things and the heaven is the air or wind where the sun, the moon, and the stars hang shining. There are two kinds of air--the heaven-air and the earth-air. The motion of the heavens is contrary to that of running water. The heavens move from east to west, while water runs from west to east. In some districts, indeed, water in the earth runs towards the north, but meets the earth-air which obstructs its flow, causes much agitation, and finally its complete evaporation from the surface of the earth. The vapour thus formed ascends and becomes clouds, which are again turned into rain by the action of the wind. The water has periods of increase and decrease according to the male and female seasons; thus in summer, which is the male season, water increases, while in winter, or the female season, it diminishes. Again, the earth-air is changed into rain when it moves from east to west ; and therefore, previous to rain, we see a white vapour in the morning ascending in the east. "This is a clear proof of the earth's growing hot." For the same reason mountains become somewhat darker just before rain.

Thunder is produced by the mingling of the male and female principles. Sounds are of ten heard in the earth in the neighbourhood of volcanoes. This is due to the 